\title{
Designing Health Care Applications for the Deaf
}

\author{
Paolo Prinetto, Gabriele Tiotto \\ Dipartimento di Automatica e Informatica \\ Politecnico di Torino \\ Turin, Italy \\ \{Paolo.Prinetto, gabriele.tiotto\}@polito.it
}

\author{
Andrea Del Principe \\ Centro Ricerche ed Innovazione Tecnologica \\ RAI \\ Turin, Italy \\ a.falletto@rai.it
}

\begin{abstract}
This paper addresses the topic of the inclusion of deaf people in the health care context. It discusses the results of recent researches developed in the design of an application for the automatic translation from the Italian to the Italian Sign Language of the deaf. The system allows the translation and visualization of the signs through a virtual avatar with the aim of improving the service quality and the accessibility of healthcare structures. This work proposes a system under development to be fully portable as a support of the ICT infrastructure or as a web service in pervasive health applications.
\end{abstract}

Sign-Language Synthesis; Virtual Signing; E-inclusion; Accessibility; Automatic Translation

\section{INTRODUCTION}

Inclusion of deaf people is a significant step towards the improvement of healthcare services. In the United States hearing impairment is the second most prevalent chronic condition. Hearing impaired constitutes the $9 \%$ of the population in the US and are increasing with the increase of the aging of the population with a perspective of growing faster than the total US population between 1980 and 2050 [1]. Recent studies targeting the relationship between deaf and the English National Health Service (NHS), report that deaf people suffer of unacceptable difficulties when they try to use the NHS services [2]. Furthermore older surveys such as the 1977 National Health Service Interview Survey, underline that the hearing-impaired people uses the healthcare services more frequently than the hearing people, feeling their health condition as poorer that the hearing-people one. The motivation of this barrier between the health care institutions and the deaf people has to be found in the difficulties hearing impaired find in communicating with the hospital staff. The $77 \%$ of British Sign Language users that visit a hospital could not easily communicate with the staff [2]. These results demonstrate that this lead to a significant decrease in the hospital efficiency especially during emergency visits.

\section{A HEALTHCARE ENVIRONMENT FOR THE DEAF}

The interpretation of these results leads to the consideration that an application that could facilitate the communication between the deaf people and the doctor is a straightforward solution. It could improve the hospital efficiency by dropping down the cost in terms of wasted time for deaf and hearing people and in terms of waste of money spent by the structure to provide an interpreter. Integrating a translation module in a healthcare and pervasive healthcare environment could be ap- plied to different scenarios. For example a Hospital structure deaf oriented with terminals that provide information translated into sign language and healthcare applications that could provide communication between the deaf and the staff in the hospital or at home. The first requires a design strongly service based while the second targets rehabilitation and therapy.

\section{AN ITALIAN TO ISL TRANSLATION SYSTEM}

Recent studies targeting sign language translation brought to the development of application for the translation of American Sign Language. The state of the art is actually the Sign Smith platform [3]. However a complete translation according to the ASL is not performed. In the application design field efforts have been made to cover the needs of universal accessibility of software in respect of blind population [4]. Other works explore accessibility of software user interfaces to improve accessibility to deaf individuals that imply the use of sign languages [5]. Despite what it is widely expected, deaf people that uses sign languages as their primary form of communication can't read written Italian sentences since the grammar of Italian Sign Language (ISL) is different from the spoken language one. Moreover reading capability of average deaf adults doesn't exceed the Primary School age. For these reasons an application deaf-oriented should translate the Italian written text into the written ISL and display the signs from their language by means of a virtual character. The use of video sequences to display a signing interpreter is more accepted by the deaf people as ensures a perfect sign performance but is not reusable solution and needs a too wide band transmission. Past research project in this field demonstrated that a signing avatar could be accepted by the deaf and brought to encouraging results. In a healthcare environment the terminals should display the results of the translation as an avatar in a window near the written text.

The application should take into account of the peculiarities of the ISL. Below we summarize the main characteristics of the ISL. Each sign can be identified by 5 parameters: Configuration (the hand shape), Orientation (the orientation of the palm), Place (where the sign is performed), Movement, Nonmanual components (facial expressions, body movements...). Since the first four of them are required to form a sign, we need to find a source of information in which these parameters are contained. Another important characteristic of ISL is the presence of classifiers. They are used to express the relationship between objects when they are signed on the body. For

The work presented in the present paper has been developed within the ATLAS (Automatic Translation into sign LAnguageS) Project, co-funded by Regione Piemonte within the "Converging Technologies - CIPE 2007" framework (Research Sector : Cognitive Science and ICT). 


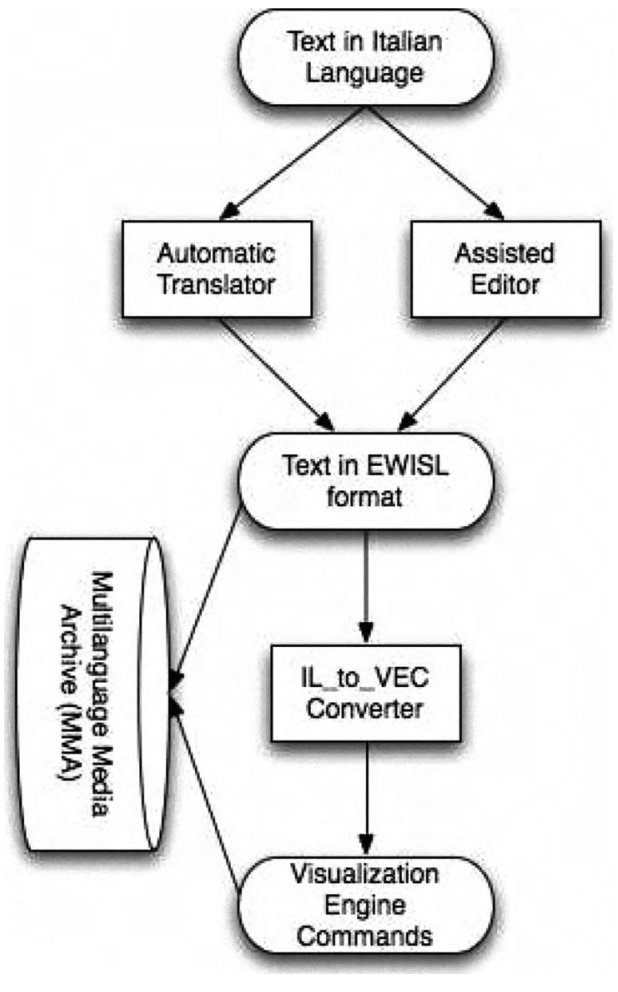

Figure 1. System Architecture

example to sign "the dog follows the cat" the signer performs the sign of "dog" with the right hand, then performs the classifier of "dog" which is another sign that expresses the canine animal. Then signs the "cat" and then the classifier for feline animals both with the left hand. Then performs the first classifier that follows in the space the second. Another characteristic of the ISL is what we call the "positional information" that allows the virtual character to position an apple (performed with a hand) on a table (performed with the other hand) signing the sentence "the apple is on the table". The information about classifiers and positional information should enrich the representation of the text to be translated, as it is not explicitly specified in the sentence. Italian deaf refers to an Italian to ISL Dictionary [6]. This is considered as a standard-de-facto by the deaf community and contains a Notation System that codes a sign with custom symbols. For each sign the Radutzky Notation (RN) codes each parameter with one or more symbols giving a formal description of the sign. In our system the RN is properly coded and stored in a database. This can be considered as a Multilanguage Media Archive (MMA) containing the basic information of the sign and constitutes the ground layer for any application deaf-oriented. It also contains movies showing the signs preformed by a true interpreter. The output of the translation modules provides an Extended Written Italian Sign Language (EWISL) format, which is a translation in written ISL, enriched with annotation and peculiarities of the ISL grammar. It allows also specifying the classifiers and positional information we have mentioned above and is expressed by means of a formal grammar such as the BackusNaur Form. It is representative enough to catch all the sign language peculiarities and in the same time contains important information to parameterize the avatar movements. The IL_to_VEC Converter converts the EWISL to commands for the graphic engine, in order to parameterize the avatar movements. The commands should be implemented in a language in the procedural animation domain such as markup languages (VRML, BALM or the more sign language oriented SiGML [7]). This could produce lightweight parameters to be sent to the avatar in a web application. After the elaboration, the MMA will contain the Italian word, the corresponding EWISL and the commands for that word produced by the IL_to_VEC. The MMA constitutes an ISL database that allows translation, storage and insertion of new signs through a custom user interface. The deaf user can take advantage of this architecture by opening a browser on the hospital terminals in order to retrieve services information translated with the described application. The virtual character plugin will provide the translation of the content that would normally be available for hearing users.

\section{CONCLUSION}

This paper describes the design guidelines for the development of an Italian to ISL translation system. The system could be applicable on the healthcare environment to support the communication between deaf people and the medical staff and could improve the accessibility of the deaf community to the health system structures. This system has been studied and is under development in the ATLAS project funded by the Piedmont Region in Italy with the purpose to be deployed to several platforms such as Web, Digital Terrestrial Television and Mobile.

\section{REFERENCE}

[1] P. Zazove, L. C. Niemann, D. W. Gorenflo, C. Carmack, D. Mehr, J. C. Coyne, T. Antonucci, "The Health Status and Health Care Utilization of Deaf and Hard-of-Hearing Persons", in Archives of Family Medicine, Vol. 7, n. 2, 1993, pp. 745-752.

[2] RNID: A simple cure: UK report on deaf and hard of hearing people's experiences of the National Health Service. National Health Service library http://www.library.nhs.uk/ENT/ViewResource.aspx?resID=263515

[3] VCom3D website: http://www.vcom3d.com/

[4] Stephanidis, C., Savidis, A., "Universal access in the information society: methods, tools, and interaction technologies", Univ. Access Inf. Soc., vol. 1, 2001, pp. 40-55.

[5] S. Fotinea, E. Efthimiou, G. Caridakis, K. Karpouzis, "A knowledgebased sign synthesis architecture", Univers. Access Inf. Soc., vol. 6, 2008, pp. 405-418.

[6] Mason Perkins Deafness Fund. Dizionario Bilingue Elementare della Lingua dei Segni Italiana LIS. Kappa, Rome, Italy, 2001. (In Italian)

[7] R. Elliott, J. R. W. Glauert, V. Jennings, and J.R. Kennaway, "An Overview of the SiGML Notation and SiGMLSigning Software System," in 4th International Conference on Language Resources and Evaluation (LREC 2004)., O. Streiter and C. Vettori, Lisbon, Portugal, 2005, pp. 98-104.

The work presented in the present paper has been developed within the ATLAS (Automatic Translation into sign LAnguageS) Project, co-funded by Regione Piemonte within the "Converging Technologies - CIPE 2007" framework (Research Sector : Cognitive Science and ICT). 